AJH

ISSN : 0973-4767
Article history : Received : 09.07.2020 Revised : 02.11 .2020 Accepted : 16.11 .2020

\section{Evaluation of chemical changes in karonda candy during storage at ambient temperature}

\author{
Ashwini B. Dinde, P. S. Joshi ${ }^{1}$, S.A. Tayade ${ }^{1}$ and S.V. Bondre ${ }^{1}$
}

\begin{abstract}
The studies were based on effect of different recipes on storage period of Karonda candy. The experiment comprised of two types of karonda i.e. green and pink with seed and without seed were stored at ambient temperature. From the findings it was observed that, there was a gradual increase in TSS, acidity, reducing sugars and total sugars content of candy irrespective of fruit colour type and recipes used in experimentation. However, $\mathrm{pH}$, nonreducing sugars, ascorbic acid, moisture, iron content of candy were found to be decreased with the advancement of storage period. The karonda candy prepared from different recipes of sugar concentration of $60 \mathrm{~B}, 65 \mathrm{~B}$ and $70 \mathrm{~B}$ can be stored upto 120 days without any microbial count. From the present findings it is concluded that karonda candy with 65 Brix sugar is suitable sugar concentration for karonda candy preparation.
\end{abstract}

KEY WORDS : Karonda candy, Sugar concentrations, Green fruits, Pink fruits

HOW TO CITE THIS ARTICLE : Dinde, Ashwini B., Joshi, P.S., Tayade, S.A. and Bondre, S.V. (2020). Evaluation of chemical changes in karonda candy during storage at ambient temperature. Asian J. Hort., 15(2) : 26-33, DOI : 10.15740/HAS/TAJH/15.2/26-33. Copyright@2020: Hind Agri -Horticultural

Society
Author for correspondence : Ashwini B. Dinde

Commercial Fruit Nursery Unit, College of Horticulture, Dr. Panjabra Deshmukh Krishi Vidyapeeth, Akola (M.S.) India

Email : psjoshihort@gmail.com
$\mathrm{F}$ ruits, whether fresh or dried, have always formed a part of the staple diet of human beings because they are rich in nutrients and provide the essential minerals and vitamins. Karonda (Carissa carandas Linn.) is well known as a protective hedge plant yielding berry like fruits are edible and attractive in colour. It is indigenous fruit of India and belongs to family apocynacae. Karonda is very hardy evergreen bush growing well even on marginal and inferior land when most other fruits either fail to grow or give poor performance. In India it was cultivated by the Europeans in kitchen garden to get fruit for jelly preparation. Karonda is usually valued for its important nutritional qualities and also recognized as richest source of carbohydrates, protein, fat, potassium and iron. Karonda fruit usually contains- 83.67 per cent moisture, 2.3 per cent protein, 4.7 per cent carbohydrates. 6 per cent minerals, 1.5 per cent fibre, 0.021 per cent calcium, 0.028 per cent phosphorus, 9-11 mg ascorbic acid (mg/100g). Karonda is widely used medicinal plant by tribal throughout India. It is cultivated in kitchen gardens farmer $\times \mathrm{s}$ fields and orchards as hedge plant. Under the changing world trade scenario, it is exploited on a commercial scale as a fruit for the processing industries. The fruits are astringent and slightly acidic in taste, it also contain maximum amount of pectin, vitamins and minerals. Due to astringent in taste of fruit, no one can eat this fruit without processing. Ones the fruit processed. The availability of processed product is possible throughout the year. The different processed products could be possibly prepared from karonda fruit such as chutney, pickle, jam, jelly, squash, beverage which has a 
great demand in national and international market considering its cost of production, which is also very less due to its maximum availability of fruits for processing as a raw material. Preservation of fruits is the more important due to non-availability of fresh produce throughout the year. Considering the nutritional and medicinal importance of karonda fruit crop, its candy can be prepared and preserved. The present investigation was carried out to find the effect of different sugar concentrations on quality of karonda candy and to find out the suitable concentration of sugar and maximum limit of storage period of karonda candy to obtain maximum consumer acceptability.

\section{RESEARCH METHODS}

The experiment was conducted in Post Harvest Technology Laboratory, Dr. PDKV, Akola, during the academic year 2017-18. Candy prepared from green and pink type fruit with or without seed. For the experimentation, matured green and pink colour type fruits with uniform sized were procured from AICRP ON Citrus, Dr. PDKV, Akola. Approximately $20 \mathrm{~kg}$ matured karonda fruits was brought to the laboratory and sorted out. Ripe, diseased, damaged and off type fruits were strictly discarded. The selected fruits were thoroughly washed with clean water to remove dirt. For candy with seed, whole fruits were used and candy without seed the fruits were cut into halves and seeds were removed.

\section{Treatment details:}

Factor A (Fruit type) :

$\mathrm{F}_{1}$ - Green colour fruit

$\mathrm{F}_{2}-$ Pink colour fruit

Factor B (Recipes) :

$\mathrm{T}_{1}$ - Fruit pieces without seed impregnated with sugar having TSS 60 Brix.

$\mathrm{T}_{2}-$ Fruit pieces without seed impregnated with sugar having TSS 65 Brix.

$\mathrm{T}_{3}$ - Fruit pieces without seed impregnated with sugar having TSS 70 Brix.

$\mathrm{T}_{4}$ - Whole fruit with seed impregnated with sugar having TSS 60 Brix.

$\mathrm{T}_{5}$ - Whole fruit with seed impregnated with sugar having TSS 65 Brix.

$\mathrm{T}_{6}-$ Whole fruit with seed impregnated with sugar having TSS 70 Brix.

\section{Preparation of karonda candy:}

Healthy and good quality matured unriped fruits were selected for candy preparation. For experimentation pink with whole fruit with seed and fruit pieces without seed are used. Then whole fruit and pieces were impregnated with sharp stainless steel fork and then cut into equal sizes slices, seeds and hard cores were removed. The prepared slices as well as whole fruits of Karonda then blanched in $500 \mathrm{ppm}$ potassium metabisulphite, 0.25 per cent citric acid and 2 per cent salt solution with hot water treatments to become the slices and whole fruit soft. Then these prepared slices and fruit were steeped in syrup of 60,65 and 70 Brix with the addition of sugar at different concentrations. The sugar syrup initially prepared at concentration of 40 Brix. After 24 hours of steeping in each treatment, the syrup concentration was increased by adding sugar proportionally. The syrup concentration was increased by 5 Brix every time until the concentration reached upto 60,65 and 70 Brix according to the treatment for a period until the equilibrium was reached between slices and the syrup concentration. Finally, the pieces and whole fruit as per treatment impregnated in each treatment were drained free of syrup and rinse immediately with tap water and dried in shade for $24 \mathrm{hrs}$. After drying, the candy was packed in 250 gauge polythene packets and those kept in plastic boxes which stored under ambient condition. The chemical observations were recorded at every 30 days interval for 120 days.

\section{RESEARCH FINDINGS AND DISCUSSION}

The changes in $\mathrm{pH}(\%)$, total soluble solids ( $\left.{ }^{\circ} \mathrm{Brix}\right)$, acidity $(\%)$, ascorbic acid $(\mathrm{mg} / 100 \mathrm{~g})$, reducing sugars $(\%)$, non-reducing sugars $(\%)$, total sugars $(\%)$ at 120 days of storage as influenced by different fruit types and recipes on $\mathrm{pH}$ of karonda candy at ambient storage is presented in Table 1 and 3.

pH (\%):

Effect of fruit types :

Data presented in Table 1 about $\mathrm{pH}$ of karonda candy were gradually decreased in both types of fruit.

\section{Effect of recipes :}

Data indicated in Table 1 during the storage period's $\mathrm{pH}$ content of karonda candy were gradually decreased in all recipes under study. At initial days, maximum $\mathrm{pH}$ was found in treatment $\mathrm{T}_{3}(3.73 \%)$ and results were 
found significant at 30,60,90, 120 days of storage. At 120 days of storage significantly maximum $\mathrm{pH}$ was recorded in $\mathrm{T}_{3}(3.43)$ and minimum in treatment $\mathrm{T}_{2}(3.25)$ but at par with treatment $\mathrm{T}_{5}(3.30)$.

\section{Interaction effects :}

The data revealed that, the interaction effect of fruit type and recipes were significant at initial to 30 days of storage. However at 60, 90 and 120 days of storage the
$\mathrm{pH}$ was found non-significant. At initial days of storage maximum $\mathrm{pH}$ was observed in treatment combination $\mathrm{F}_{1} \mathrm{~T}_{3}(3.86 \%)$. At 120 days of storage maximum $\mathrm{pH}$ was recorded in $\mathrm{F}_{1} \mathrm{~T}_{3}(3.50 \%)$. The decrease in $\mathrm{pH}$ value of karonda candy with an increase in storage period might be due to the overall increase in acidity of stored candy. Decrease in $\mathrm{pH}$ value during storage was also reported by Wani et al. (2013) in karonda jam and Zeeshan et al. (2017) in dhakki dates candy.

\begin{tabular}{|c|c|c|c|c|c|c|c|c|c|c|c|c|c|c|c|}
\hline \multirow[t]{2}{*}{ Treatments } & \multicolumn{5}{|c|}{$\mathrm{pH}$} & \multicolumn{5}{|c|}{ TSS (?rix) } & \multicolumn{5}{|c|}{ Acidity (\%) } \\
\hline & \multicolumn{15}{|c|}{ Factor A (Fruit type) } \\
\hline Storage (days) & 0 & 30 & 60 & 90 & 120 & 0 & 30 & 60 & 90 & 120 & 0 & 30 & 60 & 90 & 120 \\
\hline $\mathrm{F}_{1}$ & 3.72 & 3.65 & 3.60 & 3.50 & 3.38 & 69.35 & 72.37 & 74.43 & 75.28 & 76.28 & 1.07 & 1.09 & 1.12 & 1.12 & 1.13 \\
\hline$F_{2}$ & 3.56 & 3.50 & 3.47 & 3.39 & 3.31 & 69.03 & 69.76 & 73.66 & 74.39 & 75.57 & 1.17 & 1.19 & 1.21 & 1.22 & 1.22 \\
\hline F test & Sig & Sig & Sig. & Sig. & Sig. & Sig. & Sig & Sig. & Sig. & Sig. & Sig. & Sig. & Sig. & Sig. & Sig. \\
\hline S.E. \pm & 0.018 & 0.017 & 0.012 & 0.013 & 0.012 & 0.078 & 0.146 & 0.036 & 0.114 & 0.018 & 0.008 & 0.009 & 0.005 & 0.004 & 0.002 \\
\hline \multirow[t]{2}{*}{ C.D. $(\mathrm{P}=0.05)$} & 0.053 & 0.049 & 0.035 & 0.038 & 0.035 & 0.230 & 0.429 & 0.106 & 0.335 & 0.052 & 0.025 & 0.026 & 0.015 & 0.012 & 0.006 \\
\hline & \multicolumn{15}{|c|}{ Factor B (Recipe) } \\
\hline $\mathrm{T}_{1}$ & 3.68 & 3.60 & 3.55 & 3.41 & 3.35 & 65.14 & 70.05 & 73.92 & 74.56 & 75.91 & 1.02 & 1.03 & 1.05 & 1.07 & 1.07 \\
\hline $\mathrm{T}_{2}$ & 3.70 & 3.66 & 3.61 & 3.50 & 3.25 & 64.39 & 69.68 & 74.05 & 75.08 & 76.59 & 1.06 & 1.08 & 1.10 & 1.12 & 1.12 \\
\hline $\mathrm{T}_{3}$ & 3.73 & 3.68 & 3.56 & 3.46 & 3.43 & 68.61 & 71.68 & 76.10 & 76.54 & 76.94 & 1.05 & 1.07 & 1.09 & 1.10 & 1.11 \\
\hline $\mathrm{T}_{4}$ & 3.66 & 3.61 & 3.61 & 3.51 & 3.43 & 68.20 & 69.53 & 71.71 & 72.83 & 74.88 & 1.19 & 1.20 & 1.23 & 1.23 & 1.24 \\
\hline $\mathrm{T}_{5}$ & 3.55 & 3.45 & 3.41 & 3.38 & 3.30 & 71.56 & 72.11 & 73.32 & 74.57 & 75.68 & 1.19 & 1.21 & 1.23 & 1.23 & 1.24 \\
\hline $\mathrm{T}_{6}$ & 3.53 & 3.46 & 3.46 & 3.40 & 3.31 & 72.26 & 73.34 & 75.18 & 75.43 & 76.25 & 1.23 & 1.25 & 1.27 & 1.27 & 1.28 \\
\hline F test & Sig & Sig. & Sig. & Sig. & Sig. & Sig. & Sig. & Sig. & Sig. & Sig. & Sig. & Sig. & Sig. & Sig. & Sig. \\
\hline S.E. \pm & 0.031 & 0.029 & 0.020 & 0.023 & 0.020 & 0.136 & 0.253 & 0.063 & 0.198 & 0.031 & 0.014 & 0.015 & 0.009 & 0.007 & 0.005 \\
\hline \multirow[t]{2}{*}{ C.D. $(\mathrm{P}=0.05)$} & 0.092 & 0.085 & 0.060 & 0.066 & 0.060 & 0.398 & 0.743 & 0.184 & 0.581 & 0.091 & 0.042 & 0.044 & 0.027 & 0.021 & 0.014 \\
\hline & \multicolumn{15}{|c|}{ Interaction effect $F^{*} T$} \\
\hline $\mathrm{F}_{1} \mathrm{~T}_{1}$ & 3.83 & 3.76 & 3.63 & 3.46 & 3.36 & 65.52 & 71.55 & 74.44 & 75.06 & 75.91 & 1.01 & 1.02 & 1.04 & 1.05 & 1.06 \\
\hline $\mathrm{F}_{2} \mathrm{~T}_{1}$ & 3.52 & 3.43 & 3.46 & 3.36 & 3.33 & 64.76 & 68.56 & 73.40 & 74.05 & 74.50 & 1.03 & 1.05 & 1.07 & 1.08 & 1.08 \\
\hline $\mathrm{F}_{1} \mathrm{~T}_{2}$ & 3.80 & 3.76 & 3.66 & 3.53 & 3.26 & 64.23 & 68.89 & 74.33 & 75.50 & 77.01 & 1.01 & 1.02 & 1.04 & 1.05 & 1.05 \\
\hline $\mathrm{F}_{2} \mathrm{~T}_{2}$ & 3.60 & 3.56 & 3.56 & 3.46 & 3.23 & 64.55 & 70.47 & 73.77 & 74.67 & 76.17 & 1.12 & 1.14 & 1.16 & 1.18 & 1.18 \\
\hline $\mathrm{F}_{1} \mathrm{~T}_{3}$ & 3.86 & 3.80 & 3.66 & 3.56 & 3.50 & 68.62 & 75.37 & 76.82 & 76.99 & 77.58 & 1.00 & 1.05 & 1.07 & 1.09 & 1.09 \\
\hline $\mathrm{F}_{2} \mathrm{~T}_{3}$ & 3.60 & 3.56 & 3.46 & 3.36 & 3.36 & 68.59 & 68.60 & 75.39 & 76.09 & 76.29 & 1.09 & 1.10 & 1.11 & 1.12 & 1.13 \\
\hline $\mathrm{F}_{1} \mathrm{~T}_{4}$ & 3.76 & 3.70 & 3.70 & 3.53 & 3.46 & 68.62 & 70.15 & 72.25 & 73.20 & 75.05 & 1.14 & 1.16 & 1.18 & 1.19 & 1.20 \\
\hline $\mathrm{F}_{2} \mathrm{~T}_{4}$ & 3.56 & 3.53 & 3.53 & 3.50 & 3.40 & 67.78 & 68.91 & 71.16 & 72.47 & 74.71 & 1.24 & 1.25 & 1.27 & 1.27 & 1.28 \\
\hline $\mathrm{F}_{1} \mathrm{~T}_{5}$ & 3.56 & 3.46 & 3.43 & 3.43 & 3.36 & 73.12 & 73.17 & 73.41 & 74.46 & 75.79 & 1.10 & 1.12 & 1.14 & 1.13 & 1.13 \\
\hline $\mathrm{F}_{2} \mathrm{~T}_{5}$ & 3.53 & 3.43 & 3.40 & 3.33 & 3.23 & 74.00 & 74.05 & 73.23 & 74.68 & 75.58 & 1.29 & 1.31 & 1.33 & 1.34 & 1.34 \\
\hline $\mathrm{F}_{1} \mathrm{~T}_{6}$ & 3.54 & 3.44 & 3.50 & 3.46 & 3.33 & 74.29 & 75.12 & 75.33 & 76.47 & 76.37 & 1.19 & 1.21 & 1.23 & 1.24 & 1.24 \\
\hline $\mathrm{F}_{2} \mathrm{~T}_{6}$ & 3.53 & 3.50 & 3.43 & 3.33 & 3.30 & 74.23 & 74.55 & 75.04 & 75.38 & 76.25 & 1.27 & 1.29 & 1.31 & 1.31 & 1.32 \\
\hline F test & Sig & Sig. & NS & NS & NS & Sig. & Sig. & Sig. & Sig. & Sig. & Sig & Sig. & Sig. & Sig & Sig. \\
\hline S.E. \pm & 0.044 & 0.041 & 0.029 & 0.032 & 0.029 & 0.192 & 0.358 & 0.089 & 0.280 & 0.044 & 0.020 & 0.021 & 0.013 & 0.010 & 0.005 \\
\hline C.D. $(\mathrm{P}=0.05)$ & 0.129 & 0.120 & - & - & - & 0.563 & 1.051 & 0.261 & 0.822 & 0.129 & 0.060 & 0.063 & 0.038 & 0.029 & 0.014 \\
\hline
\end{tabular}


Total soluble solids (Brix):

Effect of fruit types:

The total soluble solids of karonda candy were gradually increased in all fruit types under study. At initial days of storage condition significantly maximum TSS (69.35 Brix) was found in treatment $F_{1}$. Similar trends was found at 30, 60, 90 and 120 days of storage.

\section{Effect of recipes:}

The total soluble solids of karonda candy were the increased rapidly upto $30^{\text {th }}$ days of storage and thereafter the gradual increase was observed in $60^{\text {th }}$ to $90^{\text {th }}$ and $120^{\text {th }}$ days and in storage period as compare to earlier trend.

\section{Interaction effects:}

An interaction effect of fruit color types and recipes on total soluble solids content of karonda candy was found significant at initial to 120 days of storage. At initial days maximum TSS was recorded in treatment combination

\begin{tabular}{|c|c|c|c|c|c|c|c|c|c|c|c|c|c|c|c|}
\hline \multirow{3}{*}{$\begin{array}{l}\text { Treatments } \\
\text { Storage (days) }\end{array}$} & \multicolumn{5}{|c|}{ TSS/acid ratio } & \multicolumn{5}{|c|}{ Reducing sugar (\%) } & \multicolumn{5}{|c|}{ Non-reducing sugars (\%) } \\
\hline & \multicolumn{15}{|c|}{ Factor A (Fruit type) } \\
\hline & 0 & 30 & 60 & 90 & 120 & 0 & 30 & 60 & 90 & 120 & 0 & 30 & 60 & 90 & 120 \\
\hline $\mathrm{F}_{1}$ & 64.48 & 66.12 & 66.71 & 66.96 & 67.65 & 29.15 & 30.83 & 33.21 & 37.36 & 39.78 & 28.40 & 27.73 & 26.79 & 25.76 & 25.67 \\
\hline $\mathrm{F}_{2}$ & 58.88 & 58.83 & 61.25 & 61.27 & 62.06 & 28.06 & 29.99 & 32.41 & 35.66 & 38.27 & 27.63 & 27.05 & 25.60 & 24.87 & 24.18 \\
\hline F test & Sig. & Sig. & Sig. & Sig. & Sig. & Sig. & Sig. & Sig. & Sig. & Sig. & Sig. & Sig. & Sig. & Sig. & Sig. \\
\hline S.E. \pm & 0.435 & 0.505 & 0.312 & 0.261 & 0.111 & 0.024 & 0.150 & 0.045 & 0.038 & 0.052 & 0.031 & 0.026 & 0.053 & 0.024 & 0.393 \\
\hline \multirow[t]{2}{*}{ C.D. $(P=0.05)$} & 1.278 & 1.482 & 0.917 & 0.766 & 0.325 & 0.070 & 0.440 & 0.131 & 0.111 & 0.152 & 0.091 & 0.076 & 0.155 & 0.071 & 1.155 \\
\hline & \multicolumn{15}{|c|}{ Factor B (Recipe) } \\
\hline $\mathrm{T}_{1}$ & 63.89 & 67.52 & 69.91 & 69.72 & 69.97 & 28.91 & 30.56 & 32.85 & 36.44 & 39.64 & 29.05 & 28.26 & 27.13 & 25.93 & 25.93 \\
\hline $\mathrm{T}_{2}$ & 60.50 & 64.40 & 67.24 & 67.30 & 68.63 & 29.25 & 31.32 & 33.25 & 36.97 & 40.17 & 28.91 & 29.71 & 27.08 & 26.00 & 25.94 \\
\hline $\mathrm{T}_{3}$ & 65.48 & 66.83 & 69.56 & 69.20 & 69.24 & 28.90 & 30.80 & 32.73 & 36.95 & 39.77 & 28.42 & 27.35 & 26.08 & 25.13 & 25.05 \\
\hline $\mathrm{T}_{4}$ & 57.12 & 57.80 & 58.32 & 58.97 & 60.46 & 27.39 & 28.50 & 31.84 & 35.66 & 37.69 & 27.04 & 26.21 & 25.52 & 24.75 & 22.99 \\
\hline $\mathrm{T}_{5}$ & 60.70 & 59.61 & 59.74 & 60.56 & 61.40 & 28.19 & 30.23 & 32.95 & 35.97 & 38.01 & 27.02 & 25.95 & 25.13 & 24.68 & 24.58 \\
\hline $\mathrm{T}_{6}$ & 58.39 & 58.70 & 59.10 & 58.94 & 59.41 & 28.99 & 31.05 & 33.26 & 37.06 & 38.88 & 27.64 & 26.87 & 26.22 & 25.38 & 25.05 \\
\hline F test & Sig. & Sig. & Sig. & Sig. & Sig. & Sig. & Sig. & Sig. & Sig. & Sig. & Sig. & Sig. & Sig. & Sig. & NS \\
\hline S.E. \pm & 0.754 & 0.874 & 0.541 & 0.452 & 0.192 & 0.041 & 0.260 & 0.077 & 0.065 & 0.090 & 0.054 & 0.045 & 0.091 & 0.042 & 0.681 \\
\hline \multirow[t]{2}{*}{ C.D. $(\mathrm{P}=0.05)$} & 2.214 & 2.567 & 1.589 & 1.327 & 0.563 & 0.121 & 0.763 & 0.227 & 0.192 & 0.263 & 0.158 & 0.132 & 0.269 & 0.124 & - \\
\hline & \multicolumn{15}{|c|}{ Interaction effect $F^{*} T$} \\
\hline $\mathrm{F}_{1} \mathrm{~T}_{1}$ & 64.88 & 70.15 & 71.21 & 71.06 & 71.39 & 29.76 & 31.07 & 33.50 & 37.47 & 40.38 & 29.39 & 28.88 & 27.52 & 26.43 & 26.42 \\
\hline $\mathrm{F}_{2} \mathrm{~T}_{1}$ & 62.91 & 64.88 & 68.61 & 68.38 & 68.56 & 28.07 & 30.05 & 32.20 & 35.41 & 38.91 & 28.72 & 27.63 & 26.74 & 25.44 & 25.43 \\
\hline $\mathrm{F}_{1} \mathrm{~T}_{2}$ & 63.60 & 67.34 & 71.25 & 71.68 & 72.88 & 29.87 & 32.11 & 33.90 & 38.15 & 41.35 & 29.34 & 28.82 & 27.67 & 26.34 & 26.57 \\
\hline $\mathrm{F}_{2} \mathrm{~T}_{2}$ & 57.41 & 61.46 & 63.23 & 62.93 & 64.37 & 28.63 & 30.52 & 32.60 & 35.79 & 38.99 & 28.48 & 30.60 & 26.50 & 25.66 & 25.31 \\
\hline $\mathrm{F}_{1} \mathrm{~T}_{3}$ & 68.39 & 71.44 & 71.59 & 71.63 & 70.96 & 30.10 & 32.17 & 33.33 & 38.80 & 42.11 & 29.82 & 28.76 & 27.56 & 26.19 & 26.03 \\
\hline $\mathrm{F}_{2} \mathrm{~T}_{3}$ & 62.57 & 62.82 & 67.52 & 67.76 & 67.52 & 27.70 & 29.43 & 32.13 & 35.11 & 37.44 & 27.01 & 25.95 & 24.60 & 24.07 & 24.06 \\
\hline $\mathrm{F}_{1} \mathrm{~T}_{4}$ & 59.85 & 60.48 & 60.89 & 61.17 & 62.55 & 27.39 & 27.93 & 31.75 & 35.63 & 37.87 & 26.86 & 26.44 & 25.85 & 24.83 & 24.62 \\
\hline $\mathrm{F}_{2} \mathrm{~T}_{4}$ & 54.38 & 55.13 & 55.76 & 56.76 & 58.37 & 27.40 & 29.07 & 31.94 & 35.70 & 37.50 & 27.22 & 25.98 & 25.19 & 24.68 & 21.36 \\
\hline $\mathrm{F}_{1} \mathrm{~T}_{5}$ & 64.92 & 64.97 & 65.41 & 65.52 & 66.68 & 28.62 & 30.51 & 32.83 & 36.14 & 38.04 & 27.35 & 26.38 & 25.45 & 25.06 & 25.05 \\
\hline $\mathrm{F}_{2} \mathrm{~T}_{5}$ & 54.17 & 54.25 & 55.07 & 55.60 & 56.13 & 27.76 & 29.94 & 33.08 & 35.79 & 37.98 & 26.69 & 25.51 & 24.80 & 24.31 & 24.12 \\
\hline $\mathrm{F}_{1} \mathrm{~T}_{6}$ & 62.25 & 61.93 & 60.91 & 61.67 & 61.70 & 29.16 & 31.18 & 33.97 & 37.97 & 38.93 & 27.63 & 27.09 & 26.69 & 25.70 & 25.33 \\
\hline $\mathrm{F}_{2} \mathrm{~T}_{6}$ & 54.33 & 54.79 & 57.28 & 57.54 & 57.76 & 28.82 & 30.92 & 32.55 & 36.14 & 38.82 & 27.66 & 26.65 & 25.75 & 25.87 & 24.78 \\
\hline F test & Sig & NS & Sig. & Sig & Sig. & Sig. & Sig. & Sig. & Sig. & Sig. & Sig. & Sig. & Sig. & Sig. & NS \\
\hline S.E. \pm & 1.066 & 1.237 & 0.765 & 0.639 & 0.271 & 0.058 & 0.367 & 0.109 & 0.092 & 0.127 & 0.076 & 0.064 & 0.129 & 0.060 & 0.964 \\
\hline C.D. $(\mathrm{P}=0.05)$ & 3.131 & - & 2.247 & 1.877 & 0.796 & 0.171 & 1.079 & 0.321 & 0.271 & 0.372 & 0.223 & 0.187 & 0.380 & 0.175 & - \\
\hline
\end{tabular}


$\mathrm{F}_{1} \mathrm{~T}_{6}(74.29$ Brix) and was found significantly higher than all other treatments. At 120 days minimum TSS was recorded in treatment combination $\mathrm{F}_{2} \mathrm{~T}_{1}$ (74.50 Brix) and maximum TSS in $\mathrm{F}_{1} \mathrm{~T}_{3}$ (77.58 Brix) and was found significantly higher than all other treatments but at par with $\mathrm{F}_{1} \mathrm{~T}_{2}$ (77.01 Brix). During storage increase in total soluble solids might be due to conversion of polysaccharides into sugars during hydrolysis process. Increase in TSS might also be attributed to the reduction in moisture content of the product with the advancement of storage. Increase in TSS with storage was also reported by Manivasagan et al. (2006) in karonda candy and Patil et al. (2014) in karonda candy.

\section{Acidity (\%):}

Effect of fruit types:

The acidity was found increases gradually with advancement of storage period. At initial days of storage maximum acidity was recorded in treatment $\mathrm{F}_{2}(1.17 \%)$ and found significantly higher than treatment $\mathrm{F}_{1}(1.07 \%)$

\begin{tabular}{|c|c|c|c|c|c|c|c|c|c|c|c|c|c|c|c|}
\hline \multirow{3}{*}{$\begin{array}{l}\text { Treatments } \\
\text { Storage (days) }\end{array}$} & \multicolumn{5}{|c|}{ Total su gars $(\%)$} & \multicolumn{5}{|c|}{ Ascorbic acid (mg/100g) } & \multicolumn{5}{|c|}{ Iron $(\mathrm{mg} / 100 \mathrm{~g})$} \\
\hline & \multicolumn{15}{|c|}{ Factor A (Fruit type) } \\
\hline & 0 & 30 & 60 & 90 & 120 & 0 & 30 & 60 & 90 & 120 & 0 & 30 & 60 & 90 & 120 \\
\hline $\mathrm{F}_{1}$ & 57.55 & 58.75 & 60.00 & 63.12 & 66.81 & 4.32 & 3.93 & 2.82 & 2.38 & 2.07 & 6.12 & 6.09 & 6.08 & 6.05 & 6.04 \\
\hline $\mathrm{F}_{2}$ & 55.69 & 57.04 & 58.01 & 60.53 & 64.34 & 8.02 & 7.67 & 6.58 & 5.83 & 4.69 & 6.18 & 6.14 & 6.13 & 6.11 & 6.09 \\
\hline F test & Sig. & Sig. & Sig. & Sig. & Sig. & Sig. & Sig. & Sig. & Sig. & Sig. & Sig. & Sig. & Sig. & Sig. & Sig. \\
\hline S.E. \pm & 0.039 & 0.033 & 0.085 & 0.045 & 0.394 & 0.017 & 0.017 & 0.013 & 0.012 & 0.007 & 0.002 & 0.004 & 0.003 & 0.004 & 0.002 \\
\hline \multirow[t]{2}{*}{ C.D. $(\mathrm{P}=0.05)$} & 0.114 & 0.098 & 0.251 & 0.132 & 1.158 & 0.049 & 0.050 & 0.039 & 0.035 & 0.020 & 0.007 & 0.012 & 0.008 & 0.010 & 0.007 \\
\hline & \multicolumn{15}{|c|}{ Factor B (Recipe) } \\
\hline $\mathrm{T}_{1}$ & 57.97 & 58.82 & 59.98 & 62.38 & 65.57 & 5.89 & 5.51 & 4.33 & 3.77 & 3.10 & 6.14 & 6.11 & 6.10 & 6.08 & 6.07 \\
\hline $\mathrm{T}_{2}$ & 58.16 & 61.03 & 60.34 & 62.97 & 66.11 & 6.02 & 5.69 & 4.48 & 3.96 & 3.32 & 6.16 & 6.13 & 6.12 & 6.10 & 6.08 \\
\hline $\mathrm{T}_{3}$ & 57.32 & 58.15 & 58.81 & 62.08 & 64.82 & 6.06 & 5.77 & 4.69 & 4.03 & 3.29 & 6.15 & 6.11 & 6.10 & 6.08 & 6.06 \\
\hline $\mathrm{T}_{4}$ & 54.43 & 55.27 & 57.37 & 60.42 & 60.68 & 6.58 & 6.11 & 5.01 & 4.38 & 3.56 & 6.14 & 6.11 & 6.10 & 6.08 & 6.06 \\
\hline $\mathrm{T}_{5}$ & 55.21 & 56.18 & 58.08 & 60.65 & 62.60 & 6.45 & 6.013 & 4.91 & 4.38 & 3.54 & 6.15 & 6.12 & 6.12 & 6.09 & 6.07 \\
\hline $\mathrm{T}_{6}$ & 56.64 & 57.92 & 59.48 & 62.44 & 63.93 & 6.05 & 5.70 & 4.56 & 4.12 & 3.48 & 6.15 & 6.11 & 6.10 & 6.07 & 6.06 \\
\hline F test & Sig. & Sig. & Sig. & Sig. & Sig. & Sig. & Sig. & Sig. & Sig. & Sig. & Sig. & NS & Sig. & Sig. & Sig. \\
\hline S.E. \pm & 0.067 & 0.058 & 0.148 & 0.078 & 0.683 & 0.073 & 0.030 & 0.023 & 0.020 & 0.012 & 0.004 & 0.007 & 0.005 & 0.006 & 0.004 \\
\hline \multirow[t]{2}{*}{ C.D. $(P=0.05)$} & 0.198 & 0.169 & 0.434 & 0.229 & 2.005 & 0.213 & 0.087 & 0.067 & 0.060 & 0.034 & 0.012 & - & 0.014 & 0.018 & 0.012 \\
\hline & \multicolumn{15}{|c|}{ Interaction effect $\mathbf{F} * \mathbf{T}$} \\
\hline $\mathrm{F}_{1} \mathrm{~T}_{1}$ & 59.15 & 59.96 & 61.02 & 63.90 & 66.81 & 3.98 & 3.60 & 2.48 & 2.12 & 1.66 & 6.14 & 6.11 & 6.10 & 6.08 & 6.06 \\
\hline $\mathrm{F}_{2} \mathrm{~T}_{1}$ & 56.79 & 57.69 & 58.94 & 60.86 & 64.34 & 7.81 & 7.41 & 6.19 & 5.42 & 4.54 & 6.15 & 6.12 & 6.10 & 6.09 & 6.08 \\
\hline $\mathrm{F}_{1} \mathrm{~T}_{2}$ & 59.21 & 60.93 & 61.57 & 64.49 & 67.92 & 4.29 & 4.15 & 2.90 & 2.10 & 1.92 & 6.13 & 6.10 & 6.09 & 6.07 & 6.05 \\
\hline $\mathrm{F}_{2} \mathrm{~T}_{2}$ & 57.11 & 60.93 & 59.11 & 61.46 & 64.31 & 7.75 & 7.23 & 6.07 & 5.82 & 4.72 & 6.20 & 6.17 & 6.15 & 6.13 & 6.12 \\
\hline $\mathrm{F}_{1} \mathrm{~T}_{3}$ & 59.93 & 61.13 & 60.89 & 64.99 & 68.14 & 4.15 & 3.89 & 2.74 & 2.33 & 1.99 & 6.12 & 6.08 & 6.07 & 6.05 & 6.03 \\
\hline $\mathrm{F}_{2} \mathrm{~T}_{3}$ & 54.72 & 55.38 & 56.73 & 59.18 & 61.51 & 7.97 & 7.66 & 6.64 & 5.73 & 4.59 & 6.19 & 6.15 & 6.14 & 6.11 & 6.10 \\
\hline $\mathrm{F}_{1} \mathrm{~T}_{4}$ & 54.25 & 55.49 & 57.60 & 60.46 & 62.50 & 4.89 & 4.13 & 3.12 & 2.90 & 2.33 & 6.13 & 6.09 & 6.08 & 6.05 & 6.03 \\
\hline $\mathrm{F}_{2} \mathrm{~T}_{4}$ & 54.62 & 55.05 & 57.14 & 60.39 & 58.87 & 8.26 & 8.09 & 6.89 & 5.86 & 4.74 & 6.18 & 6.13 & 6.13 & 6.11 & 6.09 \\
\hline$F_{1} T_{5}$ & 55.97 & 56.90 & 58.29 & 61.20 & 63.09 & 4.76 & 4.20 & 3.11 & 2.70 & 2.31 & 6.13 & 6.08 & 6.09 & 6.06 & 6.05 \\
\hline $\mathrm{F}_{2} \mathrm{~T}_{5}$ & 54.46 & 55.46 & 57.88 & 60.10 & 62.11 & 8.14 & 7.82 & 6.71 & 6.06 & 4.81 & 6.19 & 6.15 & 6.16 & 6.10 & 6.10 \\
\hline $\mathrm{F}_{1} \mathrm{~T}_{6}$ & 56.80 & 58.28 & 60.66 & 63.68 & 64.26 & 3.88 & 3.60 & 2.54 & 2.15 & 2.23 & 6.12 & 6.07 & 6.06 & 6.05 & 6.04 \\
\hline $\mathrm{F}_{2} \mathrm{~T}_{6}$ & 56.48 & 57.57 & 58.30 & 61.21 & 63.60 & 8.22 & 7.79 & 6.58 & 6.09 & 4.73 & 6.19 & 6.15 & 6.14 & 6.14 & 6.09 \\
\hline F test & Sig. & Sig. & Sig. & Sig. & NS & Sig. & Sig. & Sig. & Sig. & Sig. & Sig. & Sig. & Sig. & Sig. & Sig. \\
\hline S.E. \pm & 0.095 & 0.081 & 0.209 & 0.110 & 0.966 & 0.103 & 0.042 & 0.032 & 0.029 & 0.017 & 0.006 & 0.010 & 0.007 & 0.009 & 0.006 \\
\hline C.D. $(\mathrm{P}=0.05)$ & 0.280 & 0.239 & 0.614 & 0.324 & - & 0.302 & 0.124 & 0.095 & 0.085 & 0.049 & 0.017 & 0.029 & 0.020 & 0.025 & 0.017 \\
\hline
\end{tabular}

$\mathrm{NS}=$ Non-significant 
similarly $\mathrm{F}_{2}$ recorded maximum acidity at $30,60,90$ and 120 days of storage.

\section{Effect of recipes:}

At initial days of storage maximum acidity was recorded in treatment $\mathrm{T}_{6}(1.23 \%)$ while, minimum acidity was recorded in $\mathrm{T}_{1}(1.02 \%)$. Similar results were recorded at 30, 60, 90 and 120 days of storage. At 120 days of storage maximum acidity was found in treatment $\mathrm{T}_{6}(1.28 \%)$ but at par with treatment $\mathrm{T}_{5}(1.24 \%)$ while, minimum acidity was recorded by treatment $\mathrm{T}_{1}(1.07$ $\%)$.

\section{Interaction effects:}

An interaction effect of fruit type and recipes on acidity per cent of karonda candy was found significant at initial to 120 days of storage. At initial days of storage maximum acidity was recorded by treatment combination $\mathrm{F}_{2} \mathrm{~T}_{5}(1.29 \%)$. Similar results were recorded at 30,60,90 and 120 days of storage. At 120 days of storage maximum acidity was recorded in treatment combination $\mathrm{F}_{2} \mathrm{~T}_{5}(1.34 \%)$ and was significantly higher than all other treatments but at par with $\mathrm{F}_{2} \mathrm{~T}_{6}(1.32 \%)$ while, minimum acidity was recorded by $\mathrm{F}_{1} \mathrm{~T}_{1}(1.06 \%)$. Increase in acidity of karonda candy might be due to conversion of sugars into acids during the 120 days of storage. An increase in acidity with storage period has also been observed by Manivasagan et al. (2006) and Patil et al. (2014) in karonda candy.

\section{TSS/Acid ratio:}

\section{Effect of fruit types:}

The TSS /Acid ratio of karonda candy was found increased with advancement of storage period at 0 to 120 days of storage. The effect of fruit colour types on TSS/Acid ratio at 0 days to 120 days of storage period was found significant.

\section{Effect of recipes :}

At initial days of storage maximum TSS/Acid ratio was recorded by $\mathrm{T}_{3}(65.48)$ and minimum $\mathrm{TSS} /$ Acid ratio was found in treatment $T_{4}(57.12)$. Similar trend was found in 30 days of storage. At 60 days of storage maximum TSS/Acid was found in treatment $\mathrm{T}_{1}(69.91)$ and similar trend was in 90 days of storage. At 120 days of storage maximum TSS/Acid ratio was recorded by treatment $\mathrm{T}_{1}(69.97)$ but at par with treatment $\mathrm{T}_{3}(69.24)$ and minimum by treatment $\mathrm{T}_{6}(59.41)$.
Interaction effects:

An interaction effect of fruit types and recipe methods was found to be significant at initial to 120 days of storage. At initial days of storage maximum TSS/Acid recorded by treatment combination $\mathrm{F}_{1} \mathrm{~T}_{3}$ (68.39). Similar trend was found in 30 and 60 days of storage. At 90 days of storage maximum TSS/Acid ratio was found in $\mathrm{F}_{1} \mathrm{~T}_{2}$ (71.68) and minimum in $\mathrm{F}_{2} \mathrm{~T}_{5}$ (55.07). At 120 days of storage maximum TSS/Acid ratio was observed in treatment combination $\mathrm{F}_{1} \mathrm{~T}_{2}(72.88)$ but at par with $\mathrm{F}_{1} \mathrm{~T}_{1}$ (71.39) and minimum in $\mathrm{F}_{2} \mathrm{~T}_{5}$ (56.13). Increased TSS/ Acid ratio might be due to increased sugar level in karonda candy during storage period. An increase in TSS/ Acid ratio during storage period was also found by Sharma et al. (1998) in apple candy and Radhika et al. (2016) in ginger candy.

\section{Reducing sugars (\%):}

\section{Effect of fruit types :}

In general there was an increase in reducing sugars during the storage. At initial days of storage maximum reducing sugars was recorded in treatment $F_{1}(29.15 \%)$ and also similar trend was found at 30, 60, 90 and 120 days of storage where $F_{1}$ recorded significantly maximum reducing sugars than treatment $\mathrm{F}_{2}$.

\section{Effect of recipes :}

There was trend of increase in reducing sugars per cent of all recipes during storage of 120 days. At 120 days of storage maximum reducing sugars per cent was found in treatment $\mathrm{T}_{2}(29.25 \%)$ which was significantly higher than all other treatments.

\section{Interaction effects :}

At initial days of storage maximum reducing sugars was found in treatment combination $\mathrm{F}_{1} \mathrm{~T}_{3}(30.10 \%)$ while, minimum in $\mathrm{F}_{1} \mathrm{~T}_{4}(27.34 \%)$. Similar trend was found at 30 to 120 days of storage except 60 days of storage. At 60 days of storage maximum reducing sugars per cent was recorded by treatment combination $\mathrm{F}_{1} \mathrm{~T}_{2}$ $(32.11 \%)$. At 120 days of storage maximum reducing sugars per cent was observed in treatment combination $\mathrm{F}_{1} \mathrm{~T}_{3}(42.11 \%)$ while, minimum in treatment combination $\mathrm{F}_{2} \mathrm{~T}_{3}(37.44 \%)$. The increase in reducing sugars with advancement of storage might be due to increased degree of inversion of sugars. These findings are in conformity with the results reported by Rani and Bhatia (1985) in pear candy, Mehta et al. (2005) in galgal candy and 
Verma et al. (2006) in citrus peel candy.

\section{Non reducing sugars (\%):}

Effect of fruit types:

The data presented in Table 11 in respect of nonreducing sugars as influenced by the different fruit types was found to be significant at all stages of observations. At initial days of storage maximum non-reducing sugars was found in $\mathrm{F}_{1}(28.40 \%)$ at all stages of storage and also found significantly higher than $\mathrm{F}_{2}$.

\section{Effect of recipes :}

At initial days of storage maximum non-reducing sugar was recorded by treatment $\mathrm{T}_{1}(29.05 \%)$ and was significantly higher than all other treatments. At 30 days storage maximum non reducing sugar was found in treatment $\mathrm{T}_{2}(29.71 \%)$. At 60 days storage maximum non reducing sugars was recorded in treatment $T_{1}$ $(27.13 \%)$. At 90 days of storage maximum non reducing sugars found in treatment $\mathrm{T}_{2}(26.00 \%)$. At 120 days of storage non-significant difference was recorded. The minimum non-reducing sugars was recorded in treatment $\mathrm{T}_{4}(22.99 \%)$ and maximum in treatment $\mathrm{T}_{2}(25.94 \%)$ but at par with treatment $\mathrm{T}_{1}(25.93 \%)$.

\section{Interaction effects:}

In general there was decrease in non- reducing sugar content with advancement of storage period. The non reducing sugar was found to be decreased with the advancement of storage period. These results were confirmed by Nayak et al. (2012) in aonla candy and Patil et al. (2014) in karonda candy.

\section{Total sugars (\%):}

Effect of fruit types :

At initial days of storage maximum total sugars was recorded by treatment $\mathrm{F}_{1}(57.55 \%)$ which followed by treatment $\mathrm{F}_{2}(55.69 \%)$ and similar trend was there at 30 to 120 days of storage At 120 days of storage maximum total sugars was found in treatment $\mathrm{F}_{1}(66.81 \%)$ which was significantly higher than $\mathrm{F}_{2}(64.34 \%)$.

\section{Effect of recipes :}

In general there was an increase in total sugars during storage. At initial days of storage maximum total sugar was recorded by treatment $\mathrm{T}_{2}(58.16 \%)$ but at par with treatment $\mathrm{T}_{1}(57.97 \%)$ and similar trend was found at 30,60,90 and 120 days of storage where $T_{2}$ recorded significantly maximum total sugars than all other treatments. At 120 days of storage maximum total sugar was shown by treatment $\mathrm{T}_{2}(66.11 \%)$ but at par with $\mathrm{T}_{1}$ $(65.57 \%)$ and minimum by treatment $\mathrm{T}_{4}(60.68 \%)$.

\section{Interaction effects :}

An interaction effect of fruit types and recipes methods on total sugars content of karonda candy was found significant differences at all days of storage except at 120 days of storage. At initial days of storage maximum total sugars was recorded by treatment combination $\mathrm{F}_{1} \mathrm{~T}_{3}(59.93 \%)$ but at par with treatment combination $\mathrm{F}_{1} \mathrm{~T}_{2}(59.21 \%)$. At 30 days of storage maximum total sugar was found in treatment combination $\mathrm{F}_{1} \mathrm{~T}_{3}(61.13 \%)$ but at par with $\mathrm{F}_{1} \mathrm{~T}_{2}$ $(60.93 \%)$. At 60 days of storage maximum total sugar was recorded by treatment combination $\mathrm{F}_{1} \mathrm{~T}_{2}$ $(61.57 \%)$. At 90 days of storage maximum total sugar was present in treatment combination $\mathrm{F}_{1} \mathrm{~T}_{3}(64.99 \%)$ and same results found at 120 days of storage in which maximum total sugar recorded by treatment combination $\mathrm{F}_{1} \mathrm{~T}_{3}(68.14 \%)$ but at par with treatment combination $\mathrm{F}_{1} \mathrm{~T}_{2}(67.92 \%)$. Increase in total sugars throughout the storage might be because of increased degree of inversion of sugars. These results are in conformity with the results reported by Rani and Bhatia (1985) in pear candy Patil et al., (2014) in karonda candy.

\section{Ascorbic acid (mg/100g):}

Effect of fruit types :

The data presented in Table 15. In respect of ascorbic acid content as influenced by different fruit types showed significant differences at 0 to 120 days of storage.

\section{Effect of recipes :}

In general, there was decrease in ascorbic acid content during storage. Maximum ascorbic acid at initial days of storage was found in $\mathrm{T}_{4}(6.58)$ and found significantly superior over all other treatments, but at par with treatment $T_{5}(6.45)$ and similar trend was at 30 to 120 days of storage. At 120 days of storage maximum acid was found in treatment $\mathrm{T}_{4}$ (3.56) which was significantly superior to all other treatments but at par with treatment $\mathrm{T}_{5}(3.54)$.

Interaction effects:

An interaction effect of fruit types and recipes on 
ascorbic acid content of karonda candy was found to be significant at all stages at initial to 120 days of storage. At initial days of storage maximum ascorbic acid was found in treatment combination $\mathrm{F}_{2} \mathrm{~T}_{4}$ (8.26) followed by treatment combination $\mathrm{F}_{2} \mathrm{~T}_{6}(8.22)$ but at par with $\mathrm{F}_{2} \mathrm{~T}_{5}$ (8.14). At 120 days of storage maximum ascorbic acid was recorded by treatment combination $\mathrm{F}_{2} \mathrm{~T}_{5}$ (4.81) followed by $\mathrm{F}_{2} \mathrm{~T}_{4}$ (4.74) but at par with $\mathrm{F}_{2} \mathrm{~T}_{6}$ (4.73). The ascorbic acid content decreases with advancement of storage of product. Loss of ascorbic acid content was also reported by Manivasagan et al. (2006) in karonda candy.

\section{Iron (mg/100g):}

\section{Effect of fruit types :}

At initial days of storage maximum iron content was found in treatment $\mathrm{F}_{2}(6.18)$ but at par with treatment $\mathrm{F}_{1}$ (6.12) and similar results were found at 30 to 120 days of storage. At 120 days of storage maximum iron content was recorded in treatment $\mathrm{F}_{2}(6.09)$ but at par with $\mathrm{F}_{1}$ (6.04).

\section{Effect of recipes :}

The iron content was found to be decreased gradually with increase in storage period. At initial days of storage maximum iron was found in treatment $\mathrm{T}_{2}(6.16)$ but at par with treatment $\mathrm{T}_{5}(6.15)$ and $\mathrm{T}_{3}(6.15)$. At 30 days of storage maximum iron was recorded by treatment $\mathrm{T}_{2}$ (6.13), 60 days $\mathrm{T}_{2}(6.12)$ and 90 days $\mathrm{T}_{2}$ (6.10). At 120 days of storage maximum iron content was found in treatment $\mathrm{T}_{2}(6.08)$ followed by treatment $\mathrm{T}_{5}(6.07)$ but at par with treatment $\mathrm{T}_{6}(6.06)$ and treatment $\mathrm{T}_{4}(6.06)$.

\section{Interaction effects :}

At initial days of storage maximum iron content was recorded by treatment combination $\mathrm{F}_{2} \mathrm{~T}_{2}(6.20)$ but at par with treatment combination $\mathrm{F}_{2} \mathrm{~T}_{5}(6.19)$. At 60 days of storage maximum iron was found in treatment combination $\mathrm{F}_{2} \mathrm{~T}_{2}$ (6.19) and minimum in $\mathrm{F}_{1} \mathrm{~T}_{6}(6.07)$. At 90 days of storage maximum iron was recorded by $\mathrm{F}_{2} \mathrm{~T}_{2}$ (6.13). At 120 days of storage maximum iron content was recorded by treatment combination $\mathrm{F}_{2} \mathrm{~T}_{2}(6.12)$ and followed by $\mathrm{F}_{2} \mathrm{~T}_{3}(6.10)$ but at par with $\mathrm{F}_{2} \mathrm{~T}_{6}(6.09)$. There is decrease in iron content of fruit product with advancement of storage period which was also confirmed by Wani et al. (2013) in karonda jam.

\section{Conclusion:}

Chemical parameters like TSS, titrable acidity, TSS/ acidity ratio, reducing sugars, total sugars were increased with the advancement of storage period. Minimum TSS, TSS/acidity ratio, reducing sugars and total sugars was observed in pink type than green type karonda candy and minimum acidity increase in green type than green type. The karonda candy prepared from sugar concentration of $60 \mathrm{~B}, 65 \mathrm{~B}, 70 \mathrm{~B}$ can be stored upto 120 days without any microbial count. According to overall acceptability score 65 Brix is suitable sugar concentration for karonda candy preparation.

\section{REFERENCES}

Hiremath, J.B. and Rokhade, A.K. (2012). Preparation of sapota candy. Internat. J. Food Agric. \& Veterin. Sci., 2 (1) 107-112.

Kumar, Sanjeev and Singh, I.S. (2001). Storage studies of aonla fruit products at ambient temperature J. Prog. Hort., 33 (2): 169-173.

Manivasagan, S., Rana, G.S., Kumar, Surinder and Joon, M.S. (2006). Qualitative changes in karonda (Carrissa carandas Linn.) candy during storage at room temperature. Haryana $J$. Hort. Sci., 35 (1\&2):19-21.

Mehta, Anjali, Ranote, P.S. and Bawa, A.S. (2005). Processing of Kazhi lemon (Galga) peel waste for candy making. Indian Fd. Pack., 54 : 67-74.

Nayak, P., Tandon, D.K. and Bhatt, D.K. (2012). Study on changes of nutritional and organoleptic quality of flavoured candy prepared from aonla (Emblica officinalis G.) during storage. Internat. J. Nutr. \& Metabolism, 4 (7) : 100-106.

Patil, R., Raut, U.V. and Wankhede, R. S. (2014). Studies on preparation of karonda candy. Internat. J. Process. \& Post Harvest Technol., 5 (2): 136-140.

Rani, Upasna and Bhatia, B.S. (1985). Studies on pear candy processing. Indian Fd.Pack., 29 : 40-46.

Sethi, V. (1980). Studies on preparation and storage of some semi-dry preserves (Murrabba). Ph.D.Thesis, I.A.R.I., New Delhi, India.

Tripathi, V.K., Singh, M.B. and Singh, Surjit (1988). Studies on comparative compositional changes in different preserved products of anola (Emblica officinalis Gaertn.) var. Banarasi. Indian Fd.Pack., 42 (4) : 60-66. 